\title{
Does transcutaneous electrical nerve stimulation affect pain, neuropathic pain, and sympathetic skin responses in the treatment of chronic low back pain? A randomized, placebo- controlled study
}

\author{
Elif Yakşi ${ }^{1}$, Ayşegül Ketenci ${ }^{2}$, Mehmet Barış Baslo ${ }^{3}$, and Elif Kocasoy Orhan ${ }^{3}$ \\ 'Department of Physical Medicine and Rehabilitation, Abant Izzet Baysal University, Faculty of Medicine, Bolu, Turkey \\ ${ }^{2}$ Department of Physical Medicine and Rehabilitation, Istanbul University, Istanbul Faculty of Medicine, Istanbul, Turkey \\ ${ }^{3}$ Department of Neurology, Istanbul University, Istanbul Faculty of Medicine, Istanbul, Turkey
}

Received September 13, 2020

Revised January 5, 2021

Accepted January 7, 2021

Handling Editor: Woo Seog Sim

\section{Correspondence}

Elif Yakși

Department of Physical Medicine and Rehabilitation, Abant Izzet Baysal University, Faculty of Medicine, Orüs Boulevard, No: 59, Karacasu, Bolu 14020, Turkey

Tel: +905069078505

Fax: +903742628471

E-mail: elifyaksi@hotmail.com
Background: The purpose of this study was to assess the effectiveness of transcutaneous electrical nerve stimulation (TENS) in chronic low back pain and neuropathic pain.

Methods: Seventy-four patients aged 18-65 with chronic low back pain were included in the study. Baseline measurements were performed, and patients were randomized into three groups. The first group received burst TENS (bTENS), the second group conventional TENS (cTENS), and the third group placebo TENS (pTENS), all over 15 sessions. Patients' visual analogue scale (VAS) scores were evaluated before treatment (preT), immediately after treatment (postT), and in the third month after treatment (postT3). Douleur Neuropathique 4 Questions (DN4), the Modified Oswestry Low Back Pain Disability Questionnaire (MOS), the Beck Depression Inventory (BDI), and sympathetic skin response (SSR) values were also evaluated preT and postT3.

Results: A statistically significant improvement was observed in mean VAS scores post $T$ compared to preT in all three groups. Intergroup comparison revealed a significant difference between preT and postT values, that difference being assessed in favor of bTENS at multiple comparison analysis. Although significant improvement was determined in neuropathic pain DN4 scores measured at postT3 compared to preT in all groups, there was no significant difference between the groups. No statistically significant difference was also observed between the groups in terms of MOS, BDI, or SSR values at postT3 $(P>0.05)$.

Conclusions: bTENS therapy in patients with low back pain is an effective and safe method that can be employed in short-term pain control.

Key Words: Chronic Pain; Depression; Electromyography; Low Back Pain; Neuralgia; Pain Management; Surveys and Questionnaires; Sympathetic Nervous System; Transcutaneous Electric Nerve Stimulation. (c) This is an open-access article distributed under the terms of the Creative Commons Attribution Non-Commercial License (http://creativecommons.org/licenses/by-nc/4.0/), which permits unrestricted non-commercial use, distribution, and reproduction in any medium, provided the original work is properly cited.

(c) The Korean Pain Society, 2021
Author contributions: Elif Yakşi: Investigation; Ayşegül Ketenci: Study conception; Mehmet Barış Baslo: Investigation; Elif Kocasoy Orhan: Investigation. 


\section{INTRODUCTION}

Low back pain is a widespread global public health problem and cause of disability resulting in increased medical spending and workforce losses. Eighty percent of the population experience low back pain attacks at some time in their lives. Low back pain initially exhibits a good prognosis, and $54 \%-90 \%$ of patients improve within a few months. However, recurrence develops in $24 \%-80 \%$ of patients in the first year, and chronic problems capable of causing disability are seen in a mean $20 \%$ of patients [1-3].

Chronic back pain is defined as persistence for longer than three months, and progresses together with psychosocial and functional impairment [4]. Mechanical low back pains are those deriving from anatomical structures such as the vertebral column, intervertebral disks, and soft tissue, and in which underlying inflammatory, malignant, and infectious pathologies have been excluded [5]. Mechanisms causing nociceptive and neuropathic pain are involved in the pathophysiology of low back pain [6]. Nociceptive pain derives from inflammatory responses associated with tissue injury [7], while neuropathic pain has been defined as those deriving from primary lesions, dysfunction, or temporary impairment of the peripheral or central nervous system $[8,9]$. Four percent of the adult population experiences neuropathic chronic low back pain at some time in their lives. Epidemiological studies show that the incidence of neuropathic pain in patients with low back pain ranges between $17 \%$ and $54 \%$. The most common neuropathic pain presentation is chronic lumbar radiculopathy $[6,10,11]$.

In addition to various conservative therapies such as medical therapies, physiotherapy, exercise, and complementary procedures in the treatment of low back pain, multidisciplinary approaches such as behavioral cognitive therapy and, if necessary, invasive methods, can also be employed [12,13]. Transcutaneous electrical nerve stimulation (TENS), which is frequently applied in clinical practice, is a widely employed non-invasive, easily applied, and safe method with a low side-effect profile $[14,15]$.

Various effect mechanisms have been proposed for TENS. According to Melzack and Wall's 'gate control' theory, cells in the medulla spinalis substantia gelatinosa are stimulated by nociceptive and sensory signals. The 'gate control theory' proposes that pain sensation commencing from nociceptors is prevented from being transported to the higher centers of the brain where pain is perceived. According to the theory, the stimulation of large-diameter A- $\beta$ sensory afferents activates inhibitor interneurons in the substantia gelatinose in the posterior horn of the spinal cord, thus weakening the transmission of nociceptive signals transmitted by small-diameter A- $\delta$ and $C$ fibers.
In addition, a series of cascades including endogenous opioids involving supraspinal mechanisms also play a role in pain control $[16,17]$. TENS can be applied in different forms, depending on intensity and frequency (high frequency is $>50 \mathrm{~Hz}$, and low frequency $<10 \mathrm{~Hz}$ ) [17]. Different therapeutic effects can thus be achieved by modifying the frequency, duration, and amplitude of the electric current [18]. Conventional TENS (cTENS) involves the application of a high-frequency and low-intensity current, while burst TENS (bTENS) consists of a low-frequency current applied at a high frequency in burst form [19].

The primary aim of this study was to investigate the effectiveness of TENS therapy in mechanical type low back pain, and to assess the efficacy of different methods of application on pain, neuropathic pain, functional status, and attitudes and symptoms in the light of electrophysiological findings.

\section{MATERIALS AND METHODS}

The research was planned as a prospective, randomized, single-blind, placebo-controlled study. The study was performed in compliance with the Helsinki Declaration, and approval was granted by the Istanbul University Medical Faculty Clinical Research Ethical Committee (no. 2012/1295-1167).

The patients included in the study were informed about the aim of the study, its duration, the therapeutic methods to be applied in the scope of the research, and potential problems and side-effects during follow-up, both verbally and in writing, using a 'Volunteer Information Form.' All patients agreeing to participate gave written consent by signing a 'Volunteer Consent Form.'

\section{Patients}

Seventy-four patients ( 27 female, 47 male, mean age 43.3 \pm 11.3 ) aged 18-65, presenting to the Physical Medicine and Rehabilitation Clinic between January and June 2013, with mechanical type low back pain persisting for more than three months, were invited to take part in the study. Patients aged 18-65 years with mechanical chronic low back pain persisting for longer than 12 weeks and with no underlying inflammatory or neoplastic disease were included. Participants included in the study were selected from patients with lumbar disc disease without radicular compression. Thirty-two patients had degenerative disc disease and 42 had disc herniation without radiculopathy. In order to exclude neuropathic pain caused by radiculopathy, pre-treatment conduction and needle electromyography (EMG) examinations were evaluated. Patients with 
diabetes mellitus, inflammatory disease, hypothyroidism, vitamin B12 deficiency, major depression, comorbid diseases capable of affecting sympathetic skin response (SSR), or history of major trauma or surgery to the low back region were excluded from the study. In order to achieve $\alpha$ $<0.05$ and $\beta=80 \%$, according to the visual analogue scale (VAS) scores, we calculated that 21 participants would be required for each group, as described by Topuz et al. [20].

This research was designed as a single-blind study. The participants were not informed whether they were being given the main treatment or a placebo. Patients' demographic data such as age, sex, body mass index, and occupation were recorded together with their clinical characteristics. Detailed histories were taken, and clinical examinations were performed. Existing laboratory and radiological tests were also assessed. Patients were evaluated immediately after treatment (postT) for short term effects, and the third month after treatment (postT3) for long term effects. The study CONSORT (Consolidated Standards of Reporting Trials) flow diagram is summarized in Fig. 1.

\section{Assessment methods}

1) Pain measurement

A VAS was employed to assess the severity of low back pain [21]. Patients were asked to mark a point on a 10 $\mathrm{cm}$ line, on which 0 represented no pain and 10 the worst possible pain, corresponding to the severity of their pain. Patients were asked about the mean pain level felt during the day and night-time pain before treatment, at the end of treatment, and at follow-ups in the third month. VAS was applied in the early period, in contrast to the other assessment methods employed in the study, since neurological pain mechanisms are more complex, and we anticipated that a longer time would be required until significant improvement occurred.

\section{2) Neuropathic pain evaluation}

The severity of neuropathic pain was assessed at the start of treatment and in postT3 using the Douleur Neuropathique 4 Questions (DN4) scale. DN4 consists of four questions using seven items related to symptoms, three of which are associated with neurological examination. Total scores were calculated, with scores above 4 indicating the presence of neuropathic pain [22].

\section{3) Functional status evaluation}

The Modified Oswestry Low Back Pain Disability Questionnaire (MOS) consisting of 10 questions scored 0-5 was used to assess patients' functional status. The scale investigates intensity of pain, and degree of change in pain during personal care, lifting, walking, sitting, standing, sleeping, social life, and traveling. Patients' MOS values were evaluated at the start of treatment and in postT3 [23].

\section{4) Evaluation of symptoms of depression}

The Beck Depression Inventory (BDI) was applied for the purpose of measuring physical, emotional, and cognitive signs encountered in depression, such as pessimism, irritability, fatigue, and weight loss. BDI scores are evalu-

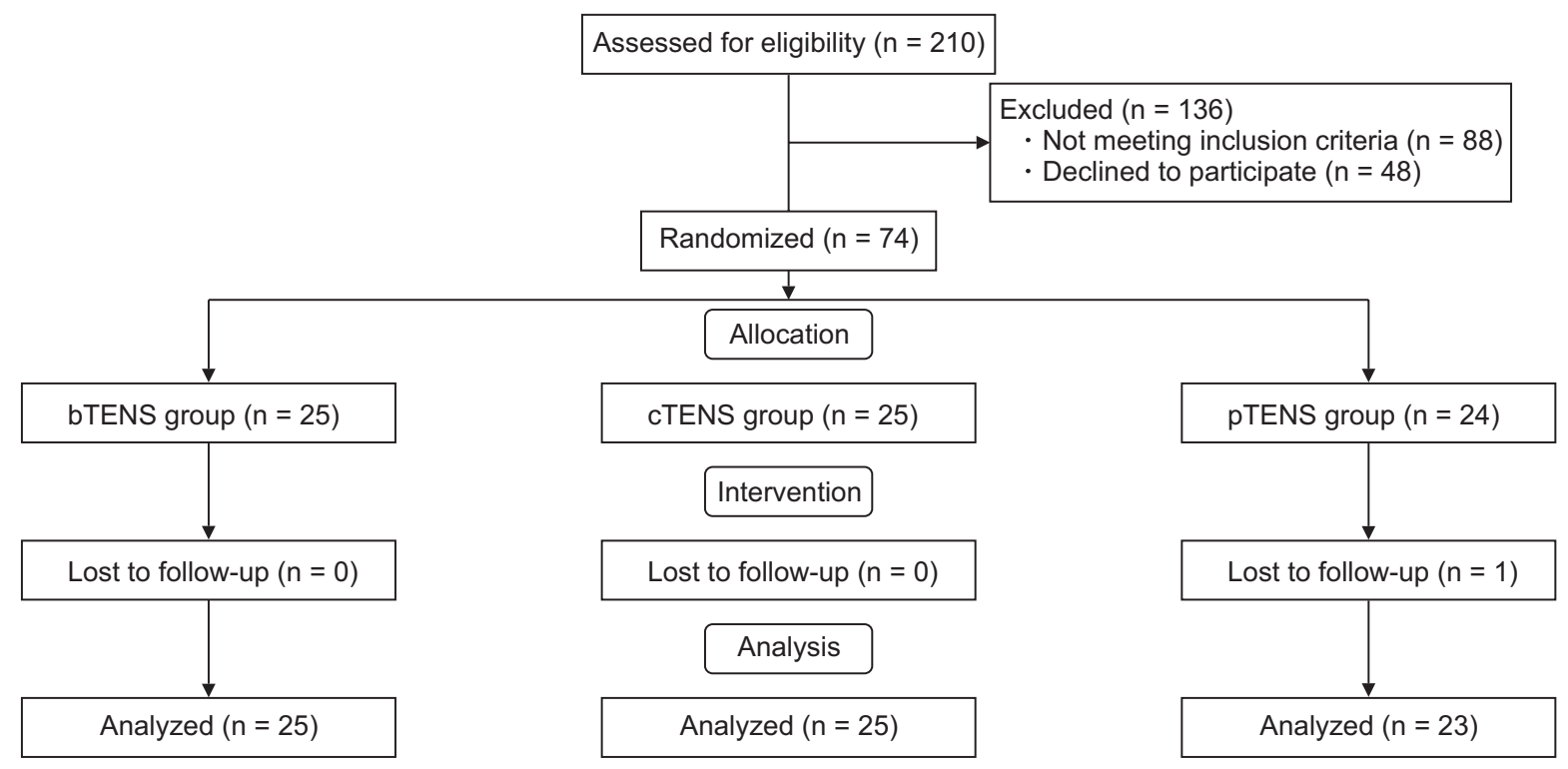

Fig. 1. CONSORT (Consolidated Standards of Reporting Trials) flow diagram of the patient inclusion process. TENS: transcutaneous electrical nerve stimulation, bTENS: burst TENS, cTENS: conventional TENS, pTENS: placebo TENS. 
ated from absence of symptoms to severe symptoms. Total scores range between 0 (no depression) to 63 (severe depression). Patients' BDI scores were evaluated at the start of treatment and in postT3 [24].

\section{5) Electromyographic evaluation}

Conduction examinations and needle EMG were performed on all patients before treatment in order to differentiate peripheral neuropathy associated with other causes and potential peripheral nerve and root lesions. SSR was subsequently studied from the hands and feet, with amplitude and latency values being recorded. SSR evaluation was performed at a room temperature of $25^{\circ} \mathrm{C}$, in a standardized, quiet, and semi-dark environment, at the Istanbul University Medical Faculty Neurology Department using an EMG device (Dantec Key Point ${ }^{\circledR}$ or twochannel Keypoint v3.03; Medtronic, Skovlunde, Denmark) permitting computer software SSR measurements. SSR was measured at a room temperature of $25^{\circ} \mathrm{C}$, with patients at rest in a supine position. Six-millimeter silver disk electrodes were employed as recording electrodes. For SSR evaluation of the upper extremity, the active (-) electrode was attached to the palmar region, the reference electrode (+) to the dorsum of the hand, and the earth electrode to the forearm. Similarly in the lower extremities, the active electrode was attached to the sole of the foot, and the reference electrode to the dorsum of the foot. SSR was obtained from the right upper and lower extremities through stimulation of the median nerve from the second finger or toe. Duration of stimulation was set to $0.1 \mathrm{msec}$ and intensity to $15-20 \mathrm{~mA}$. Stimuli were applied at varying intervals and with $>30$ seconds between stimuli in order to avoid habituation. A maximum of five unilateral stimulations were applied to each patient. SSR was regarded as unresponsive if no response was achieved to these five stimuli. Latency was defined as the time to the start of the first deflection from the start of the stimulus artefact (msec), and amplitude was measured from peak to peak $(\mathrm{mV})$. The shortest latency and highest amplitude values were included in the analysis. SSR was re-evaluated postT3.

\section{Treatment method}

Patients were divided into three groups - cTENS, bTENS, and placebo TENS (pTENS). Randomization was performed with a sequential order list using the Microsoft Excel $^{\oplus} 2003$ (Microsoft, Redmond, WA) random number production function.

TENS Intellect ${ }^{\circledR}$ Advanced (Chattanooga, Mouguerre, France) device is used in our study. cTENS stimulation parameters are $60-80 \mathrm{~Hz}$, with a pulse width of short dura- tion (50-80 $\mu \mathrm{s}), 10-30 \mathrm{~mA}$ intensity and bTENS comprises a baseline low frequency current $(1-4 \mathrm{~Hz})$ together with high frequency trains $(50-100 \mathrm{~Hz})$. Patients were informed about the therapeutic method and potential side-effects before being taken for treatment. Two channels (four electrodes), one applied to the right and the other to the left, were used in treatment. The active electrode emerging from the different channel was attached $1.5 \mathrm{~cm}$ laterally to the vertebrae at the L2-L4 level, while the passive electrode was placed $3 \mathrm{~cm}$ distally to the active electrode. TENS therapy was applied for 30 minutes in the placebo group, with no current being given to the TENS device, and at moderate intensity, depending on the patient's tolerance, in such a way as not to cause pain or discomfort, in the individuals receiving burst and conventional therapy. Patients were not given any regular medical treatment, but were permitted to use tablets containing paracetamol if required, on the condition that the dosage did not exceed $1 \mathrm{~g}$ a day. Pain diaries were given to all patients, and they were asked to register their pain intensities and paracetamol consumption at home. Treatment was applied for a total of 15 sessions, five times a week for three weeks. The placement of the electrodes in the lumbar region is shown in Fig. 2.

\section{Statistical analysis}

Statistical analysis was conducted on SPSS version 21.0 (IBM Co., Armonk, NY) software. Mean, standard deviation, median, minimum, and maximum values were calculated for all parameters.

The Kolmogorov-Smirnov and Shapiro-Wilk tests were applied on commencement to evaluate normality of distribution among the three groups. The Student $t$, Wilcoxon signed ranks, and Freidman tests were employed to compare intragroup changes. Intergroup comparisons were performed using the one-way ANOVA and Kruskal-Wallis

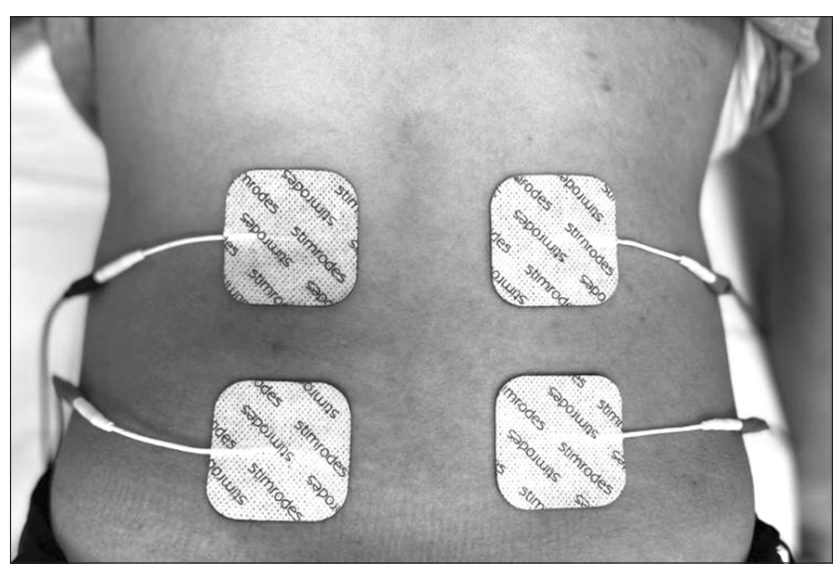

Fig. 2. Electrodes are placed in the lumbar region. 
tests. For multiple comparisons, the Mann-Whitney $U$ test with Bonferroni adjustment was used, where $P<0.05 /$ (number of comparsions) indicates a statistically significant difference between two groups. Numerical data were assessed using the chi-square test. Correlations between measurements were assessed using Spearman's rho test and the Pearson test in the light of the character and distribution of variables. Results were analyzed at a $95 \%$ confidence interval, and $P$-values $<0.05$ were regarded as statistically significant.

\section{RESULTS}

The 74 patients taking part in the study were assigned into three groups: bTENS $(n=25)$, cTENS $(n=25)$, and pTENS $(n=24)$. One patient from the placebo group was excluded from the analysis due to a failure to attend follow-ups, and the study was finally completed with 73 patients $(25$ bTENS, 25 cTENS, and 23 pTENS). Patients' demographic data and clinical characteristics are summarized in Table 1. No TENS-associated side-effect developed in any patient. No patients reported using paracetamol. Demographic and clinical characteristics were similar between

Table 1. Patients' demographic data and clinical characteristics by groups

\begin{tabular}{|c|c|c|c|c|c|}
\hline Characteristic & bTENS & cTENS & pTENS & Total & $P$ value ${ }^{a}$ \\
\hline \multicolumn{6}{|l|}{ Age } \\
\hline Mean \pm SD & $45.6 \pm 9.4$ & $43.2 \pm 12.8$ & $40.8 \pm 11.5$ & $43.3 \pm 11.3$ & 0.346 \\
\hline Median & 45.0 & 43.0 & 40.0 & 44.0 & \\
\hline Min-Max & $24.0-64.0$ & $24.0-63.0$ & $20.0-64.0$ & $20.0-64.0$ & \\
\hline \multicolumn{6}{|l|}{ Sex (\%) } \\
\hline Female & 16 (64.0) & $13(52.0)$ & $18(78.0)$ & $47(64.4)$ & 0.165 \\
\hline Male & $9(36.0)$ & $12(48.0)$ & $5(22.0)$ & $26(35.6)$ & \\
\hline \multicolumn{6}{|l|}{$\mathrm{BMI}\left(\mathrm{kg} / \mathrm{m}^{2}\right)$} \\
\hline Mean \pm SD & $28.6 \pm 4.8$ & $27.4 \pm 4.1$ & $29.9 \pm 4.6$ & $28.6 \pm 4.6$ & 0.173 \\
\hline Median & 28.1 & 26.0 & 29.3 & 28.6 & \\
\hline Min-Max & $20.5-39.5$ & $21.7-37.8$ & $22.0-40.5$ & $20.5-40.5$ & \\
\hline \multicolumn{6}{|l|}{ Occupation (\%) } \\
\hline Retired & $2(8.0)$ & $3(12.0)$ & $0(0.0)$ & $5(7.0)$ & \\
\hline Housewife & 12 (48.0) & $9(36.0)$ & $11(48.0)$ & $32(44.0)$ & \\
\hline Manual worker & $5(20.0)$ & $5(20.0)$ & $5(22.0)$ & $15(20.0)$ & \\
\hline Clerical worker & $1(4.0)$ & $1(4.0)$ & $3(13.0)$ & $5(7.0)$ & \\
\hline Student & $0(0.0)$ & $1(4.0)$ & $1(4.0)$ & $2(3.0)$ & \\
\hline Other & $5(20.0)$ & $6(24.0)$ & $3(13.0)$ & $3(4.0)$ & \\
\hline
\end{tabular}

TENS: transcutaneous electrical nerve stimulation, bTENS: burst TENS, cTENS: conventional TENS, pTENS: placebo TENS, SD: standard deviation, Min: minimum, Max: maximum, BMl: body mass index.

${ }^{a}$ Kruskal-Wallis test, analysis of variance test.

Table 2. Intragroup comparison of preT, postT, and postT3 VAS scores

\begin{tabular}{|c|c|c|c|c|c|c|}
\hline VAS scores & \multicolumn{2}{|c|}{ bTENS $(n=25)$} & \multicolumn{2}{|c|}{ cTENS $(n=25)$} & \multicolumn{2}{|c|}{ pTENS ( $n=23$ ) } \\
\hline \multicolumn{7}{|l|}{ Mean VAS scores } \\
\hline preT & $6.8 \pm 1.5$ & $7(4-10)$ & $6.2 \pm 1.7$ & $6(4-9)$ & $6.6 \pm 1.1$ & $7(5-8)$ \\
\hline postT & $2.8 \pm 2.1$ & $3(0-9)$ & $4.0 \pm 2.3$ & $4(0-9)$ & $4.5 \pm 1.6$ & $5(1-8)$ \\
\hline postT3 & $4.2 \pm 2.9$ & $4(0-10)$ & $3.7 \pm 2.9$ & $4(0-10)$ & $5.0 \pm 1.9$ & $5(1-8)$ \\
\hline$P$ value $^{a}$ & $<0.001$ & & $<0.001$ & & 0.016 & \\
\hline \multicolumn{7}{|c|}{ Night-time VAS scores } \\
\hline preT & $4.6 \pm 3.0$ & $5(0-9)$ & $4.4 \pm 3.3$ & $5(0-9)$ & $2.2 \pm 2.5$ & $6(0-8)$ \\
\hline postT & $1.8 \pm 2.4$ & $0(0-9)$ & $2.7 \pm 2.9$ & $2(0-9)$ & $2.9 \pm 3.0$ & $3(0-8)$ \\
\hline postT3 & $2.2 \pm 2.5$ & $1(0-10)$ & $3.1 \pm 2.3$ & $2(0-10)$ & $3.6 \pm 3.0$ & $4(0-8)$ \\
\hline$P$ value ${ }^{a}$ & $<0.001$ & & 0.003 & & $<0.001$ & \\
\hline
\end{tabular}

Values are presented as mean \pm standard deviation or median (minimum-maximum).

preT: before treatment, postT: after treatment, postT3: third month after treatment, VAS: visual analogue scale, TENS: transcutaneous electrical nerve stimulation, bTENS: burst TENS, cTENS: conventional TENS, pTENS: placebo TENS.

${ }^{a}$ Freidman test. $\alpha=0.05$. 



Fig. 3. Intergroup comparisons of mean and night-time visual analogue scale (VAS) scores. Asterisk (*) indicates statistically significant differences between the time points within the group. For the lines in a box and whisker plot: error bars are the $95 \%$ confidence interval, the bottom and top of the box are the 25th and 75th percentiles, the line inside the box is the 50th percentile (median), and any outliers are shown as open circles or asteriks. TENS: transcutaneous electrical nerve stimulation, bTENS: burst TENS, cTENS: conventional TENS, pTENS: placebo TENS, preT: before treatment, postT: after treatment, postT3: third month after treatment.

Table 3. Intergroup comparison of preT, postT, and postT3 VAS scores

\begin{tabular}{|c|c|c|c|c|c|c|c|}
\hline VAS scores & \multicolumn{2}{|c|}{ bTENS $(n=25)$} & \multicolumn{2}{|c|}{ cTENS $(n=25)$} & \multicolumn{2}{|c|}{ pTENS $(n=23)$} & $P$ value \\
\hline \multicolumn{8}{|l|}{ Mean VAS scores } \\
\hline postT-preT & $-3.9 \pm 2.4$ & $-4(-10-0)$ & $-2.2 \pm 1.9$ & $-2(-7-0)$ & $-2.0 \pm 1.7$ & $-2.0(-6-0)$ & 0.012 \\
\hline postT3-postT & $-2.6 \pm 2.7$ & $-3(-2-7)$ & $-0.2 \pm 2.1$ & $0(-5-3)$ & $-0.4 \pm 2.3$ & $0.0(-4-6)$ & 0.260 \\
\hline postT3-preT & $-1.3 \pm 2.7$ & $1(-2-7)$ & $-2.5 \pm 2.7$ & $-2(-9-3)$ & $-1.6 \pm 2.0$ & $-2.0(-6-3)$ & 0.358 \\
\hline \multicolumn{8}{|c|}{ Night-time VAS scores } \\
\hline postT-preT & $-2.8 \pm 2.9$ & $-2(-9-1)$ & $-1.6 \pm 1.9$ & $-1(-5-0)$ & $-2.3 \pm 2.4$ & $-2(-8-0)$ & 0.463 \\
\hline postT3-postT & $0.4 \pm 1.5$ & $0(-2-6)$ & $0.1 \pm 1.5$ & $0(-3-3)$ & $-0.4 \pm 2.3$ & $0(-5-2)$ & 0.943 \\
\hline postT3-preT & $-2.4 \pm 2.7$ & $-2(-8-1)$ & $-1.5 \pm 2.4$ & $-1(-6-2)$ & $-1.8 \pm 2.4$ & $-2(-8-6)$ & 0.569 \\
\hline
\end{tabular}

Values are presented as mean \pm standard deviation or median (minimum-maximum).

preT: before treatment, postT: after treatment, postT3: third month after treatment, VAS: visual analogue scale, TENS: transcutaneous electrical nerve stimulation, bTENS: burst TENS, cTENS: conventional TENS, pTENS: placebo TENS.

${ }^{a}$ Kruskal-Wallis test. $\alpha=0.05$.

the three groups $(P>0.05)$.

The preT, postT, and postT3 mean, standard deviation, median, and min-max values for the mean VAS and night-time VAS scores are shown in Table 2. Intragroup comparisons revealed significant improvement in mean and night-time pain severity measured using VAS in postT and postT3 mean values compared to preT (Table 2). PreT, postT, and postT3 VAS score changes are shown in Fig. 3. Intergroup comparison revealed that this significance was between postT and preT values (Table 3). A Mann-Whitney $U$ test significance level with Bonferroni correction of $P$ $<0.05 / 3(0.0167)$ was adopted. Significant differences between post $\mathrm{T}$ and preT values were observed between the bTENS and pTENS groups $(P=0.007)$. Multiple compari- sons of postT and preT VAS scores among the groups using the Mann-Whitney $U$ test (with Bonferroni correction) revealed significant improvement in favor of bTENS (Table 4).

Neuropathic pain assessment was performed on 74 patients included in the study before treatment using the DN4 scale. The 40 patients subsequently diagnosed with neuropathic pain (DN4 > 4) were then subjected to subgroup analysis based on treatment groups. PreT and postT3 mean, standard deviation, median, and min-max DN4 values are shown in Table 5. Intragroup analysis revealed statistically significant improvement in all groups in DN4 measurements in postT3 values. However, no statistically significant difference was observed in intergroup 
comparisons.

Third-month intragroup analysis of MOS values revealed significant improvement in all the treatment groups, but no statistically significant difference was observed between them (Table 6). No statistically significant improvement was observed in BDI depressive symptoms at postT3 at intragroup analyses, and no significant difference was also determined among the three groups (Table 6).

Intragroup comparisons revealed no statistically sig-

Table 4. Intergroup multiple comparison analysis of postT and preT VAS scores

\begin{tabular}{rcc}
\hline \multirow{2}{*}{ postT-preT VAS } & \multicolumn{2}{c}{ Mann-Whitney U test (Bonferroni correction) } \\
\cline { 2 - 3 } & $\mathrm{Z}$ & $P$ value \\
\hline bTENS-cTENS & -2.375 & 0.018 \\
bTENS-pTENS & -2.685 & $0.007^{\mathrm{a}}$ \\
cTENS-pTENS & -0.348 & 0.728 \\
\hline
\end{tabular}

preT: before treatment, postT: after treatment, VAS: visual analogue scale, TENS: transcutaneous electrical nerve stimulation, bTENS: burst TENS, cTENS: conventional TENS, pTENS: placebo TENS.

${ }^{a} P$ values indicate a statistical significance at $P<0.0167$, after Bonferonni adjustment. nificant difference at postT3 in terms of SSR latency and amplitude values obtained from the upper extremity, or of SSR latency values recorded from the lower extremity. Similarly, there was no statistically significant difference between the groups. Intragroup analysis of SSR amplitude values recorded from the lower extremity increased significantly in the bTENS and pTENS groups, but no significant difference was observed between the three study groups (Table 7).

\section{DISCUSSION}

This study evaluated the efficacy of TENS on pain and neuropathic pain in chronic back pain in a placebo-controlled manner. The primary outcome of this study was that bTENS treatment was significantly more effective in short-term pain control compared to cTENS and pTENS in terms of pain severity capable of measurement using VAS scores. The secondary outcome was that bTENS and cTENS treatments were no different from the placebo in terms of neuropathic pain control.

Although data exist for the short-term effectiveness of TENS therapy, which has been employed in clinical prac-

Table 5. Intra- and intergroup comparison of preT and postT3 DN4 scores

\begin{tabular}{|c|c|c|c|c|c|c|c|}
\hline DN4 scores & \multicolumn{2}{|c|}{ bTENS $(n=13)$} & \multicolumn{2}{|c|}{ cTENS $(n=14)$} & \multicolumn{2}{|c|}{ pTENS $(n=13)$} & $P$ value $^{a}$ \\
\hline preT & $5.4 \pm 1.5$ & $5(4-8)$ & $5.1 \pm 1.3$ & $4(4-8)$ & $5.0 \pm 1.1$ & $5(4-7)$ & \multirow{2}{*}{0.479} \\
\hline postT3 & $2.5 \pm 2.3$ & $2(0-7)$ & $3.4 \pm 2.2$ & $3(0-7)$ & $3.1 \pm 2.1$ & $2(0-7)$ & \\
\hline$P$ value $^{\mathrm{b}}$ & \multicolumn{2}{|c|}{$<0.001$} & \multicolumn{2}{|c|}{$<0.001$} & \multicolumn{2}{|c|}{$<0.001$} & \\
\hline
\end{tabular}

Values are presented as mean \pm standard deviation or median (minimum-maximum).

preT: before treatment, postT3: third month after treatment, DN4: Douleur Neuropathique 4 Questions, TENS: transcutaneous electrical nerve stimulation, bTENS: burst TENS, cTENS: conventional TENS, pTENS: placebo TENS.

${ }^{a}$ Kruskal-Wallis test. ${ }^{b} t$-test.

Table 6. Intra- and intergroup comparison of preT and postT3 MOS and BDI values

\begin{tabular}{|c|c|c|c|c|c|c|c|}
\hline $\begin{array}{c}\text { MOS and BDI } \\
\text { values }\end{array}$ & \multicolumn{2}{|c|}{ bTENS ( $n=25)$} & \multicolumn{2}{|c|}{ cTENS $(n=25)$} & \multicolumn{2}{|c|}{ pTENS ( $n=23$ ) } & $P$ value ${ }^{a}$ \\
\hline \multicolumn{8}{|l|}{ MOS } \\
\hline preT & $32.0 \pm 6.3$ & $31(18-45)$ & $30.6 \pm 8.2$ & $32(14-47)$ & $32.6 \pm 6.3$ & $33(18-44)$ & \multirow{2}{*}{0.476} \\
\hline postT3 & $25.4 \pm 9.4$ & $25(11-45)$ & $26.0 \pm 10.6$ & $28(10-48)$ & $28.3 \pm 7.9$ & $29(14-43)$ & \\
\hline$P$ value ${ }^{\mathrm{b}}$ & \multicolumn{2}{|c|}{0.001} & \multicolumn{2}{|c|}{0.003} & \multicolumn{2}{|c|}{0.002} & \\
\hline \multicolumn{8}{|l|}{$\mathrm{BDI}$} \\
\hline preT & $15.4 \pm 7.3$ & $16(3-29)$ & $10.0 \pm 6.1$ & $9(0-26)$ & $10.7 \pm 5.5$ & $10(1-23)$ & \multirow{2}{*}{0.283} \\
\hline postT3 & $14.2 \pm 8.5$ & $14(1-29)$ & $10.7 \pm 7.7$ & $11(0-30)$ & $9.3 \pm 6.1$ & $8(1-26)$ & \\
\hline$P$ value ${ }^{\mathrm{b}}$ & \multicolumn{2}{|c|}{0.428} & \multicolumn{2}{|c|}{0.594} & \multicolumn{2}{|c|}{0.160} & \\
\hline
\end{tabular}

Values are presented as mean \pm standard deviation or median (minimum-maximum).

preT: before treatment, postT3: third month after treatment, MOS: Modified Oswestry Low Back Pain Disability Questionnaire, BDI: Beck Depression Inventory, TENS: transcutaneous electrical nerve stimulation, bTENS: burst TENS, cTENS: conventional TENS, pTENS: placebo TENS.

${ }^{a}$ Kruskal-Wallis test, ANOVA test. ${ }^{b}$-test. 
Table 7. Intra- and intergroup comparison of preT and postT3 SSR values

\begin{tabular}{|c|c|c|c|c|c|c|c|}
\hline SSR values & \multicolumn{2}{|c|}{ bTENS $(n=25)$} & \multicolumn{2}{|c|}{ CTENS $(n=25)$} & \multicolumn{2}{|c|}{ pTENS $(n=23)$} & $P$ value \\
\hline \multicolumn{8}{|c|}{ Upper extremity } \\
\hline \multicolumn{8}{|c|}{ Latency (ms) } \\
\hline preT & $1.4 \pm 0.2$ & $1.4(1.2-1.8)$ & $1.3 \pm 0.2$ & $1.3(0.9-1.6)$ & $1.3 \pm 0.2$ & $1.4(0.8-1.8)$ & \multirow{2}{*}{0.913} \\
\hline postT3 & $1.4 \pm 0.2$ & $1.4(0.7-1.8)$ & $1.3 \pm 0.1$ & $1.3(0.9-1.6)$ & $1.4 \pm 0.1$ & $1.4(1.2-1.5)$ & \\
\hline$P$ value & \multicolumn{2}{|c|}{0.993} & \multicolumn{2}{|c|}{0.542} & \multicolumn{2}{|c|}{0.821} & \\
\hline \multicolumn{8}{|c|}{ Amplitude (mV) } \\
\hline preT & $2.7 \pm 2.6$ & $2.0(0.8-10.9)$ & $2.9 \pm 1.7$ & $1.7(0.8-7.4)$ & $2.7 \pm 1.6$ & $2.4(0.4-5.2)$ & \multirow{2}{*}{0.660} \\
\hline postT3 & $2.7 \pm 1.9$ & $1.9(0.9-8.0)$ & $2.8 \pm 1.4$ & $2.6(1.2-4.9)$ & $3.6 \pm 2.1$ & $3.0(1.2-8.4)$ & \\
\hline$P$ value & \multicolumn{2}{|c|}{0.937} & \multicolumn{2}{|c|}{0.575} & \multicolumn{2}{|c|}{0.508} & \\
\hline \multicolumn{8}{|c|}{ Lower extremity } \\
\hline \multicolumn{8}{|c|}{ Latency (ms) } \\
\hline preT & $2.1 \pm 0.3$ & $2.0(1.7-2.7)$ & $1.7 \pm 0.2$ & $1.8(1.2-2.0)$ & $1.8 \pm 0.3$ & $1.9(1.1-2.1)$ & \multirow{2}{*}{0.903} \\
\hline postT3 & $1.9 \pm 0.4$ & $1.8(1.2-2.6)$ & $1.7 \pm 0.2$ & $1.7(1.2-2.1)$ & $1.6 \pm 0.2$ & $1.7(1.2-2.0)$ & \\
\hline$P$ value & \multicolumn{2}{|c|}{0.367} & \multicolumn{2}{|c|}{0.484} & \multicolumn{2}{|c|}{0.314} & \\
\hline \multicolumn{8}{|c|}{ Amplitude (mV) } \\
\hline preT & $0.9 \pm 0.4$ & $0.8(0.3-1.7)$ & $1.1 \pm 0.6$ & $1.0(0.3-2.0)$ & $1.5 \pm 1.3$ & $0.9(0.4-4.8)$ & \multirow{2}{*}{0.275} \\
\hline postT3 & $2.0 \pm 1.6$ & $1.3(0.5-5.9)$ & $1.2 \pm 0.9$ & $0.8(0.4-2.9)$ & $2.3 \pm 1.4$ & $1.8(0.8-4.9)$ & \\
\hline$P$ value & \multicolumn{2}{|c|}{0.016} & \multicolumn{2}{|c|}{0.575} & \multicolumn{2}{|c|}{0.037} & \\
\hline
\end{tabular}

Values are presented as mean \pm standard deviation or median (minimum-maximum).

preT: before treatment, postT3: third month after treatment, SSR: sympathetic skin response, ms: millisecond, mV: millivolt, TENS: transcutaneous electrical nerve stimulation, bTENS: burst TENS, cTENS: conventional TENS, pTENS: placebo TENS.

${ }^{a}$ ANOVA. ${ }^{b}$-test. ${ }^{c}$ Wilcoxon test.

tice for many years, the data for long-term effectiveness are inconsistent [14,25-27]. Marchand et al. [28] compared the effectiveness of cTENS and pTENS in chronic low back pain. Those authors reported that cTENS application was significantly more effective than placebo in the control of early period pain, but that no difference was detected between the two therapies at the third and sixth months after treatment. Jarzem et al. [29] reported that cTENS was superior to a placebo in the control of short-term pain. Topuz et al. [20] also concluded that cTENS was more effective in the control of short-term pain than a placebo in chronic low back pain.

The superiority of cTENS over a placebo reported in these studies shows that active TENS application is effective in the control of short-term pain. Although the active TENS treatment bTENS was superior to placebo in shortterm pain control, cTENS was not superior to placebo in the control of short-term pain in the present study. This discrepancy between the present study and previous research may derive from methodological differences. The cTENS method stimulates group II (A $\beta$ b) fibers and provides analgesia through spinal segmental mechanisms providing inhibition of type IV (C) fibers and type III (A $\delta \mathrm{d}$ ) fibers. The bTENS method stimulates group III (A $\delta \mathrm{d})$ nociceptive fibers to release endogenous opiate at the spinal and supraspinal levels [30]. bTENS provides spinal analgesia, and may also provide stronger analgesia by binding to opioid receptors in the brain by releasing the body's own endorphins. While the effectiveness of TENS in chronic low back pain has previously been evaluated in conventional or acupuncture mode, to the best of our knowledge there have been no studies investigating burst, and the present research is thus the first from that perspective.

The great majority of studies involving TENS have focused on healing in the nociceptive component of pain, and very few studies have examined its effectiveness on the neuropathic component. The majority of those studies that have been conducted have considered diabetic peripheral neuropathies and trigeminal neuralgias, and have reported that TENS is effective in controlling pain in these diseases [31-35]. Forst et al. [36] compared active TENS and a placebo in the treatment of diabetes-related neuropathic pain and reported that active TENS was superior to the placebo at 6 and 12 weeks. Active TENS may have emerged as superior to the placebo on neuropathic pain scores, in contrast to the present study, due to the use in that study of a questionnaire, a subjective evaluation tool. In another study, Jin et al. [32] reported that active TENS administered to patients with diabetic peripheral neuropathy resulted in a significantly greater improvement in shortterm neuropathic pain scores compared to a placebo, but observed no statistically significant difference between the groups in the third month. The long-term results of the present research are consistent with these previous studies. In addition, due to different pathophysiology, clinical findings and healing processes involved in neuropathic 
pains, the treatment results for neuropathic pain developing in association with diabetes and low back pain are not suitable for comparison.

Of the 74 patients included in the present study, 40 had neuropathic pain detected using the DN4 scale. Epidemiological studies show that the incidence of neuropathic pain in patients with low back pain ranges between $17 \%$ and $54 \%$ [10]. The neuropathic pain rates for each group in the present study were thus consistent with the previous literature. The effect of TENS treatment on neuropathic pain being no different from that of the placebo in this study may be due to the insufficient number of patients. In addition, improvement may not have been adequately defined due to the broad spectrum of neuropathic pain symptoms and the relatively limited content of the DN4 scale compared to other scales evaluating neuropathic pain. To the best of our knowledge, very few studies have investigated the effectiveness of TENS in terms of the neuropathic pain component of low back pain [37], and the present research is the first such extensive placebo-controlled study. The absence of any neurological or systemic disease or vitamin deficiency in our patients, and the use of objective EMG examinations for assessment purposes all enhance the reliability of this study.

Numerous studies have assessed pain together with functional status, but no statistically significant improvement has been determined following TENS therapies $[20,29]$. Similarly, in the present patient group, active TENS had no statistically significant effect on functional status. Pain is known to be correlated with functional restriction [38]. The lack of a significant decrease in pain at the third month controls in the present study may have resulted in the lack of significant improvement in functional restriction.

Psychological and social dimensions may be affected in addition to the presence of physical symptoms in patients with chronic pain. The reported prevalence of major depression in patients with pain is $13 \%-85 \%$ [39]. The presence of anxiety, stress, and somatization symptoms in patients with chronic pain has been linked to pain development $[40,41]$. It should be remembered that while pain may be a symptom of depression, depressive disorders triggered by pain may also be encountered [42]. One systematic review detected no difference between the effects of a placebo and TENS applied for chronic pain control on depression scales [14]. This is also consistent with the findings of the present study. The lack of any major change in BDI values after treatment may be due to the fact that patients diagnosed with major depression were not included in the study. In addition, the exclusion of patients with major depression also made it possible to exclude pain emerging in association with depression.
Electrophysiological reflections of diseases associated with neurological involvements are highly important in terms of diagnosis, prognosis and treatment. In addition to nerve conduction studies and needle EMG, SSR assessing the autonomous nervous system reveals momentary potential changes reflecting sudomotor changes on the skin surface [43]. Although SSR has been evaluated in numerous diseases, few studies have examined the relationship between chronic low back pain and SSR. Some studies have determined abnormal sympathetic system findings in SSR measurements in patients with lumbosacral radiculopathy $[44,45]$, and these impairments have been reported to be capable of contributing to pain exhaustion and chronicization [45].

The number of studies evaluating neurophysiological responses, in terms of treatment methods employed in low back pain, is limited. Perry et al. [46] reported that active manipulation therapies directed toward the lumbar region affected simultaneous SSR measurements and increased sympathoexcitatory activities, although another study reported that active exercise and manipulation resulted in significant changes in SSR [47]. One study of the effect on SSR of active TENS and sham TENS in healthy individuals reported no change in post-application latency values, while amplitude values increased significantly in the active TENS group [48]. Similarly in the present study, while no significant change was observed after treatment in the bTENS group, a significant increase was recorded in amplitude values. In addition, although the SSR amplitude changes were detected in pTENS, no statistically significant difference was determined between active and placebo applications. Although this shows that placebo administration may be effective, the wide variability in SSR amplitudes in normal individuals means that this measurement is not always regarded as a reliable parameter [49]. The fact that, in contrast to other studies, the present research evaluated long-term results may account for the inconsistency between the findings. Moreover, no statistically significant change in SSR values may be due to the absence of a significant improvement in pain and neuropathic pain scores at third month measurements.

The principal limitation of this study is that DN4, MOS, $\mathrm{BDI}$, and SSR values were not assessed at the time when significant improvement was achieved in terms of VAS scores. Although the effects of TENS methods were no different from those of the placebo on neuropathic pain scores, the low sample size means that it is not possible to conclude that TENS treatment is not effective in neuropathic pain control. In addition, patients with lumbar discopathy or degenerative disc disease were included in the study, and, therefore, a homogeneous patient group was not established. It was also not possible to evaluate 
disease-related SSR pathologies due to the absence of a healthy control group.

In conclusion, bTENS treatment aimed at the lumbar regions in patients with chronic mechanical low back pain is an effective and safe method capable of use in early nociceptive pain control. We think that it will be useful to combine TENS therapies with other physiotherapeutic modalities in order to achieve long-term effects. We also think that further studies involving larger case numbers and permitting analysis of specific diagnostic groups for chronic low back pain should now be designed to investigate the effectiveness of TENS in neuropathic pain deriving from chronic low back pain.

\section{ACKNOWLEDGMENTS}

We would like to thank Scientific Research Projects Coordination Unit of Istanbul University for funding this study.

\section{CONFLICT OF INTEREST}

No potential conflict of interest relevant to this article was reported.

\section{FUNDING}

This study was funded by Scientific Research Projects Coordination Unit of Istanbul University (Project number: 28997).

\section{ORCID}

Elif Yakși, https://orcid.org/0000-0003-1534-8205

Ayșegül Ketenci, https://orcid.org/0000-0003-3165-7358

Mehmet Barıș Baslo, https://orcid.org/0000-0003-1551-0559

Elif Kocasoy Orhan, https://orcid.org/0000-0002-2110-4832

\section{REFERENCES}

1. Freburger JK, Holmes GM, Agans RP, Jackman AM, Darter JD, Wallace AS, et al. The rising prevalence of chronic low back pain. Arch Intern Med 2009; 169: 251-8.

2. Hoy D, Brooks P, Blyth F, Buchbinder R. The epidemiology of low back pain. Best Pract Res Clin Rheumatol 2010; 24: 76981.

3. Weiner SS, Nordin M. Prevention and management of chronic back pain. Best Pract Res Clin Rheumatol 2010; 24: 267-79.
4. Kamper SJ, Apeldoorn AT, Chiarotto A, Smeets RJ, Ostelo RW, Guzman J, et al. Multidisciplinary biopsychosocial rehabilitation for chronic low back pain: Cochrane systematic review and meta-analysis. BMJ 2015; 350: h444.

5. Will JS, Bury DC, Miller JA. Mechanical low back pain. Am Fam Physician 2018; 98: 421-8.

6. Freynhagen R, Baron R. The evaluation of neuropathic components in low back pain. Curr Pain Headache Rep 2009; 13: 185-90.

7. Morlion B. Pharmacotherapy of low back pain: targeting nociceptive and neuropathic pain components. Curr Med Res Opin 2011; 27: 11-33.

8. Treede RD, Jensen TS, Campbell JN, Cruccu G, Dostrovsky JO, Griffin JW, et al. Neuropathic pain: redefinition and a grading system for clinical and research purposes. Neurology 2008; 70: 1630-5.

9. Jensen TS, Baron R, Haanpää M, Kalso E, Loeser JD, Rice ASC, et al. A new definition of neuropathic pain. Pain 2011; 152: 2204-5.

10. Kaki AM, El-Yaski AZ, Youseif E. Identifying neuropathic pain among patients with chronic low-back pain: use of the Leeds Assessment of Neuropathic Symptoms and Signs pain scale. Reg Anesth Pain Med 2005; 30: 422-8.

11. Mehra M, Hill K, Nicholl D, Schadrack J. The burden of chronic low back pain with and without a neuropathic component: a healthcare resource use and cost analysis. J Med Econ 2012; 15: 245-52.

12. Koes BW, van Tulder MW, Thomas S. Diagnosis and treatment of low back pain. BMJ 2006; 332: 1430-4.

13. Chou R, Deyo R, Friedly J, Skelly A, Hashimoto R, Weimer M, et al. Nonpharmacologic therapies for low back pain: a systematic review for an American College of Physicians Clinical Practice Guideline. Ann Intern Med 2017; 166: 493-505.

14. Khadilkar A, Odebiyi DO, Brosseau L, Wells GA. Transcutaneous electrical nerve stimulation (TENS) versus placebo for chronic low-back pain. Cochrane Database Syst Rev 2008; 2008: CD003008.

15. Vance CG, Dailey DL, Rakel BA, Sluka KA. Using TENS for pain control: the state of the evidence. Pain Manag 2014; 4: 197-209.

16. Melzack R, Wall PD. Pain mechanisms: a new theory. Science 1965;150:971-9.

17. Sluka KA, Walsh D. Transcutaneous electrical nerve stimulation: basic science mechanisms and clinical effectiveness. J Pain 2003; 4: 109-21.

18. Moran F, Leonard T, Hawthorne S, Hughes CM, McCrumGardner E, Johnson MI, et al. Hypoalgesia in response to transcutaneous electrical nerve stimulation (TENS) depends on stimulation intensity. J Pain 2011; 12: 929-35.

19. Johnson M. Transcutaneous electrical nerve stimulation: review of effectiveness. Nurs Stand 2014; 28: 44-53.

20. Topuz O, Özfidan E, Ozgen M, Ardic F. Efficacy of trans- 
cutaneous electrical nerve stimulation and percutaneous neuromodulation therapy in chronic low back pain. J Back Musculoskelet Rehabil 2004; 17: 127-33.

21. Price DD, McGrath PA, Rafii A, Buckingham B. The validation of visual analogue scales as ratio scale measures for chronic and experimental pain. Pain 1983; 17: 45-56.

22. Bouhassira D, Attal N, Alchaar H, Boureau F, Brochet B, Bruxelle J, et al. Comparison of pain syndromes associated with nervous or somatic lesions and development of a new neuropathic pain diagnostic questionnaire (DN4). Pain 2005; 114: 29-36.

23. Hudson-Cook N, Tomes-Nicholson K, Breen A. A revised Oswestry disability questionnaire. In: Back pain: new approaches to rehabilitation and education. Edited by Roland MO, Jenner JR. Manchester, Manchester University Press. 1989, pp 187-204.

24. Hisli N. [A study on the validity of Beck depression inventory]. Psikol Derg 1988; 6: 118-22. Turkish.

25. Milne S, Welch V, Brosseau L, Saginur M, Shea B, Tugwell P, et al. Transcutaneous electrical nerve stimulation (TENS) for chronic low back pain. Cochrane Database Syst Rev 2001; 2: CD003008.

26. Brosseau L, Milne S, Robinson V, Marchand S, Shea B, Wells $\mathrm{G}$, et al. Efficacy of the transcutaneous electrical nerve stimulation for the treatment of chronic low back pain: a metaanalysis. Spine (Phila Pa 1976) 2002; 27: 596-603.

27. Khadilkar A, Milne S, Brosseau L, Robinson V, Saginur M, Shea B, et al. Transcutaneous electrical nerve stimulation (TENS) for chronic low-back pain. Cochrane Database Syst Rev 2005; 3: CD003008.

28. Marchand S, Charest J, Li J, Chenard JR, Lavignolle B, Laurencelle L. Is TENS purely a placebo effect? A controlled study on chronic low back pain. Pain 1993; 54: 99-106.

29. Jarzem PF, Harvey EJ, Arcaro N, Kaczorowski J. Transcutaneous electrical nerve stimulation [TENS] for short-term treatment of low back pain-randomized double blind crossover study of sham versus conventional TENS. J Musculoskelet Pain 2005; 13: 11-7.

30. van der Spank JT, Cambier DC, De Paepe HM, Danneels LA, Witvrouw EE, Beerens L. Pain relief in labour by transcutaneous electrical nerve stimulation (TENS). Arch Gynecol Obstet 2000; 264: 131-6.

31. Dubinsky RM, Miyasaki J. Assessment: efficacy of transcutaneous electric nerve stimulation in the treatment of pain in neurologic disorders (an evidence-based review): report of the Therapeutics and Technology Assessment Subcommittee of the American Academy of Neurology. Neurology 2010; 74: 173-6.

32. Jin DM, Xu Y, Geng DF, Yan TB. Effect of transcutaneous electrical nerve stimulation on symptomatic diabetic peripheral neuropathy: a meta-analysis of randomized controlled trials. Diabetes Res Clin Pract 2010; 89: 10-5.
33. Pieber K, Herceg M, Paternostro-Sluga T. Electrotherapy for the treatment of painful diabetic peripheral neuropathy: a review. J Rehabil Med 2010; 42: 289-95.

34. Singla S, Prabhakar V, Singla RK. Role of transcutaneous electric nerve stimulation in the management of trigeminal neuralgia. J Neurosci Rural Pract 2011; 2: 150-2.

35. Yameen F, Shahbaz NN, Hasan Y, Fauz R, Abdullah M. Efficacy of transcutaneous electrical nerve stimulation and its different modes in patients with trigeminal neuralgia. J Pak Med Assoc 2011; 61: 437-9.

36. Forst T, Nguyen M, Forst S, Disselhoff B, Pohlmann T, Pfützner A. Impact of low frequency transcutaneous electrical nerve stimulation on symptomatic diabetic neuropathy using the new Salutaris device. Diabetes Nutr Metab 2004; 17: 163-8.

37. Ghoname EA, White PF, Ahmed HE, Hamza MA, Craig WF, Noe CE. Percutaneous electrical nerve stimulation: an alternative to TENS in the management of sciatica. Pain 1999; 83: 193-9.

38. Pengel LH, Herbert RD, Maher CG, Refshauge KM. Acute low back pain: systematic review of its prognosis. BMJ 2003; 327: 323.

39. Tsang A, Von Korff M, Lee S, Alonso J, Karam E, Angermeyer $\mathrm{MC}$, et al. Common chronic pain conditions in developed and developing countries: gender and age differences and comorbidity with depression-anxiety disorders. J Pain 2008; 9: 883-91.

40. Andersson GB. Epidemiological features of chronic low-back pain. Lancet 1999; 354: 581-5.

41. Oksuz E. Prevalence, risk factors, and preference-based health states of low back pain in a Turkish population. Spine (Phila Pa 1976) 2006; 31: E968-72.

42. Kara H, Abay E. [Psychiatric approach to chronic pain]. Anadolu Psikiyatri Derg 2000; 1: 89-99. Turkish.

43. Vetrugno R, Liguori R, Cortelli P, Montagna P. Sympathetic skin response: basic mechanisms and clinical applications. Clin Auton Res 2003; 13: 256-70.

44. Erdem Tilki H, Coşkun M, Unal Akdemir N, Incesu L. Axon count and sympathetic skin responses in lumbosacral radiculopathy. J Clin Neurol 2014; 10: 10-6.

45. El-Badawy MA, El Mikkawy DM. Sympathetic dysfunction in patients with chronic low back pain and failed back surgery syndrome. Clin J Pain 2016; 32: 226-31.

46. Perry J, Green A, Singh S, Watson P. A randomised, independent groups study investigating the sympathetic nervous system responses to two manual therapy treatments in patients with LBP. Man Ther 2015; 20: 861-7.

47. Perry J, Green A. A longitudinal observational clinical study of neurophysiological and patient-reported responses to a program of physiotherapy for acute and subacute low back pain. J Manipulative Physiol Ther 2018; 41: 456-66.

48. Okuyucu EE, Turhanoğlu AD, Guntel M, Yılmazer S, Savaș N, 
Mansuroğlu A. Does transcutaneous nerve stimulation have effect on sympathetic skin response? J Clin Neurosci 2018; 47: 160-2.
49. Aramaki S, Kira Y, Hirasawa Y. A study of the normal values and habituation phenomenon of sympathetic skin response. Am J Phys Med Rehabil 1997; 76: 2-7. 